\title{
Fabrication and characterization of high aspect ratio perpendicular patterned information storage media in an $\mathrm{Al}_{2} \mathrm{O}_{3} / \mathrm{GaAs}$ substrate
}

\author{
Joyce Wonga) and Axel Scherer \\ Department of Electrical Engineering, California Institute of Technology, Pasadena, California 91125 \\ Mladen Todorovic and Sheldon Schultz \\ Center for Magnetic Recording Research and Department of Physics, University of California, San Diego, \\ La Jolla, California 92093-0319
}

\begin{abstract}
In a new approach, we have fabricated 6:1 aspect ratio magnetic nanocolumns, 60-250 $\mathrm{nm}$ in diameter, embedded in a hard aluminum-oxide/gallium-arsenide $\left(\mathrm{Al}_{2} \mathrm{O}_{3} / \mathrm{GaAs}\right)$ substrate. The fabrication technique uses the highly selective etching properties of GaAs and AlAs, and highly efficient masking properties of $\mathrm{Al}_{2} \mathrm{O}_{3}$ to create small diameter, high aspect ratio holes. Nickel (Ni) is subsequently electroplated into the holes, followed by polishing, which creates a smooth and hard surface appropriate for future reading and writing of the columns as individual bits for high density information storage. We have used magnetic force microscopy and scanning magneto-resistance microscopy to characterize the resulting magnets. We find the columns more magnetically stable than previously achieved with magnets embedded in a $\mathrm{SiO}_{2}$ substrate. Such stability is necessary before further writing of perpendicular patterned media can be demonstrated. (c) 1999 American Institute of Physics. [S0021-8979(99)63408-9]
\end{abstract}

\section{INTRODUCTION}

By combining high resolution electron beam lithography, etching, and electroplating, it is now possible to microfabricate nanometer scale magnets. Recently there has been an effort to employ such nanomagnets as individual bits for perpendicular patterned magnetic storage in order to increase the bit density for magnetic storage in the range of 20-100 Gbits/in. ${ }^{2}$ Our initial work has focused on creating freestanding magnets with lateral dimensions as small as $20 \mathrm{~nm}{ }^{1}$ More realistic structures made of embedded Ni columns in a $\mathrm{SiO}_{2}$ substrate, with aspect ratios of $4: 1$, have also been demonstrated. ${ }^{2}$ Our previous studies have shown that this aspect ratio is not sufficient to achieve the magnetic stability required for reliable reading and writing of this form of media. ${ }^{3}$ In order to achieve a sufficiently high coercivity, embedded magnets of higher aspect ratio are desirable. We have re-examined the possible substrate material selection for the lithographic production of high aspect ratio patterned media and have now developed a new and more robust fabrication procedure suitable for embedding high aspect ratio nanomagnets into a durable matrix.

Instead of using polymethyl methacrylate (PMMA) $e$-beam resist or silicon dioxide $\left(\mathrm{SiO}_{2}\right)$, we employ $\mathrm{Al}_{2} \mathrm{O}_{3}$ and GaAs. It has been found that the etch rate selectivity of $\mathrm{Al}_{2} \mathrm{O}_{3}$ over $\mathrm{GaAs}$ is larger than 30:1. ${ }^{4}$ Thus, by combining the robust $\mathrm{Al}_{2} \mathrm{O}_{3}$ mask, high resolution electron beam lithography, and the directional chemically assisted ion beam etching (CAIBE), it is now possible to achieve small diameter and high aspect ratio holes in the GaAs substrate, which are then filled with $\mathrm{Ni}$ by electrodeposition.

\section{PROCEDURE}

A schematic flow diagram of the fabrication procedures in defining the $\mathrm{Ni}$ column arrays embedded in an

\footnotetext{
${ }^{a)}$ Electronic mail: joyceyw@its.caltech.edu
}

$\mathrm{Al}_{2} \mathrm{O}_{3} / \mathrm{GaAs}$ substrate is shown in Fig. 1. A $200 \mathrm{~nm}$ layer of AlAs and a $50 \mathrm{~nm} \mathrm{GaAs} \mathrm{cap} \mathrm{layer} \mathrm{are} \mathrm{grown} \mathrm{by} \mathrm{molecular}$ beam epitaxy (MBE) on top of a GaAs substrate. A $200 \mathrm{~nm}$ layer of $\mathrm{SiO}_{2}$ is then sputter deposited and a $10 \mathrm{~nm}$ layer of $\mathrm{Cr}$ and $40 \mathrm{~nm}$ layer of $\mathrm{Au}$ are subsequently evaporated onto the sample [Fig. 1(a)]. Dot array patterns are defined on the PMMA coated sample by vector-scanned electron beam lithography, followed by development in a 3:7 cellulosemethanol mixture [Fig. 1(b)]. The patterns are transferred into the $\mathrm{Cr} / \mathrm{Au}$ layer by Ar ion milling [Fig. 1(c)] and into the $\mathrm{SiO}_{2}$ layer by $\mathrm{C}_{2} \mathrm{~F}_{6}$ reactive ion etching [Fig. 1(d)]. The $\mathrm{SiO}_{2}$

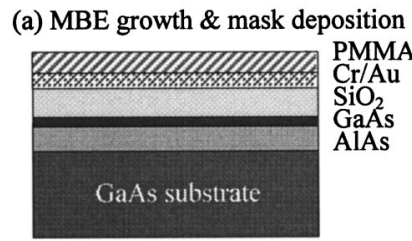

(c) Ar ion milling

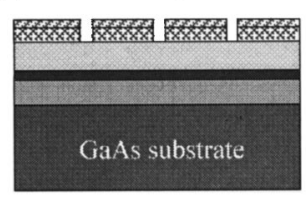

(e) CAIBE

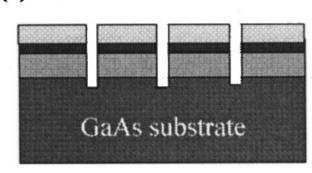

(g) CAIBE
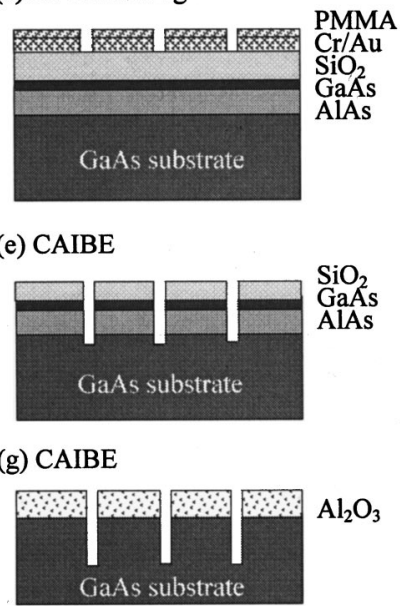

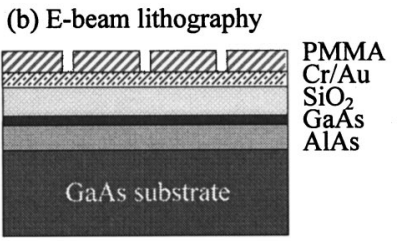

(d) $\mathrm{C}_{2} \mathrm{~F}_{6} \mathrm{RIE}$

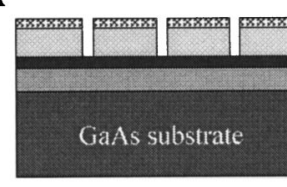

(f) Wet thermal oxidation

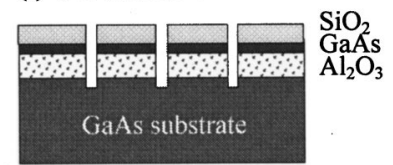

(h) Electroplating plated
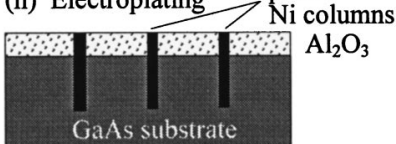

FIG. 1. Schematic diagram of the processing sequence for the fabrication of $\mathrm{Ni}$ columns embedded in an $\mathrm{Al}_{2} \mathrm{O}_{3} / \mathrm{GaAs}$ substrate. 


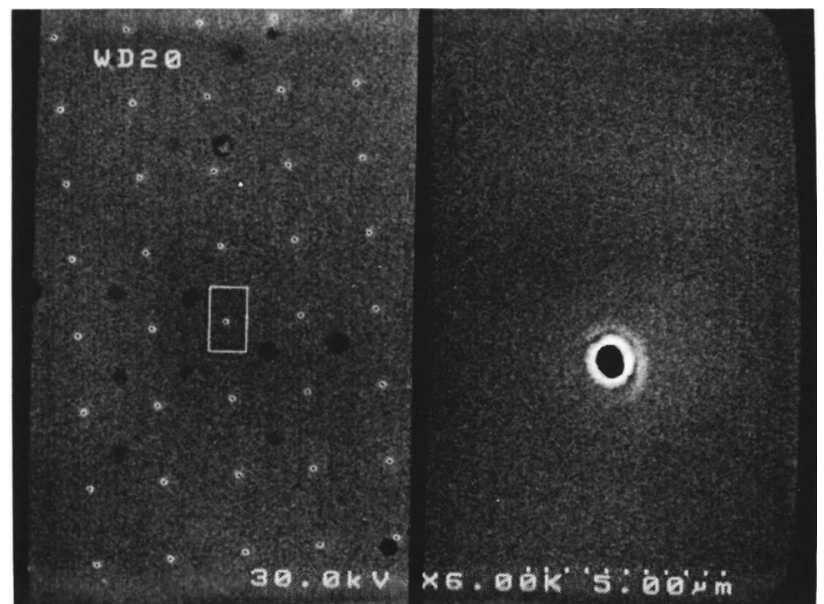

FIG. 2. SEM image of an array of etched holes (left) before filling by electroplating with $\mathrm{Ni}$, and a $10 \times$ enlargement of a single hole (right) of approximately $60 \mathrm{~nm}$ in diameter.

layer then acts as an etch mask for further pattern transfer into the AlAs layer by $\mathrm{Cl}_{2}$ assisted ion beam etching [Fig. 1(e)]. In our CAIBE system, a Kauffman $\mathrm{Ar}^{+}$ion source is used in conjunction with a gas introduction nozzle to accelerate high energy ions towards the substrate covered with an etch mask. This allows us to achieve a high etching rate and selectivity of specific materials, as well as the directionality necessary for defining high aspect ratio structures. Immediately following CAIBE, the AlAs layer is converted into $\mathrm{Al}_{2} \mathrm{O}_{3}$ by wet thermal oxidation at $380{ }^{\circ} \mathrm{C}$ for $15 \mathrm{~min}$ [Fig. 1(f) ]. ${ }^{5}$ This robust layer of $\mathrm{Al}_{2} \mathrm{O}_{3}$ is the final mask for pattern amplification in the perpendicular direction into the GaAs substrate by using further CAIBE [Fig. 1(g)]. Figure 2 shows a scanning electron microscopy (SEM) image of an array of holes (left) defined by electron beam lithography, produced by the procedures just described. In the right side of Fig. 2 , we present a $10 \times$ enlarged view of a single hole (right) of approximately $60 \mathrm{~nm}$ in diameter.

Electroplating is then used to deposit $\mathrm{Ni}$ into the holes that have been etched into the GaAs substrate [Fig. 1(h)]. The plating apparatus is identical to that in Ref. 6, where the probe contacts the sample outside of the plating bath and does not disturb the electrodeposition of the Ni columns. In our case, $\mathrm{Ni}$ is used as the anode and the conductive GaAs substrate is used as the cathode. Nickel sulfamate is used as the plating medium and a pulse current with a duty cycle of $80 \%$ ( $2 \mathrm{~s}$ on and $0.5 \mathrm{~s}$ off) is applied. The plating is conducted under an optical microscope and end point detection is possible through the change in optical contrast of the plated Ni columns. Precise end point detection is not critical in our case as any overplated $\mathrm{Ni}$ column can be polished to the desired height, which is determined by the thickness of the $\mathrm{Al}_{2} \mathrm{O}_{3}$ layer, using a mechanical polish.

Using this new approach, we have fabricated $80 \times 80$ arrays of Ni columns of $6: 1$ aspect ratio that are embedded in a hard $\mathrm{Al}_{2} \mathrm{O}_{3} / \mathrm{GaAs}$ substrate. Figure 3 shows an SEM image of an electroplated Ni dot array of $100 \mathrm{~nm}$ diameter and 2.5 $\mu \mathrm{m}$ spacing embedded in an $\mathrm{Al}_{2} \mathrm{O}_{3} / \mathrm{GaAs}$ substrate. The plating seems to be very uniform over a large area and the mechanical polishing procedure results in a clean and

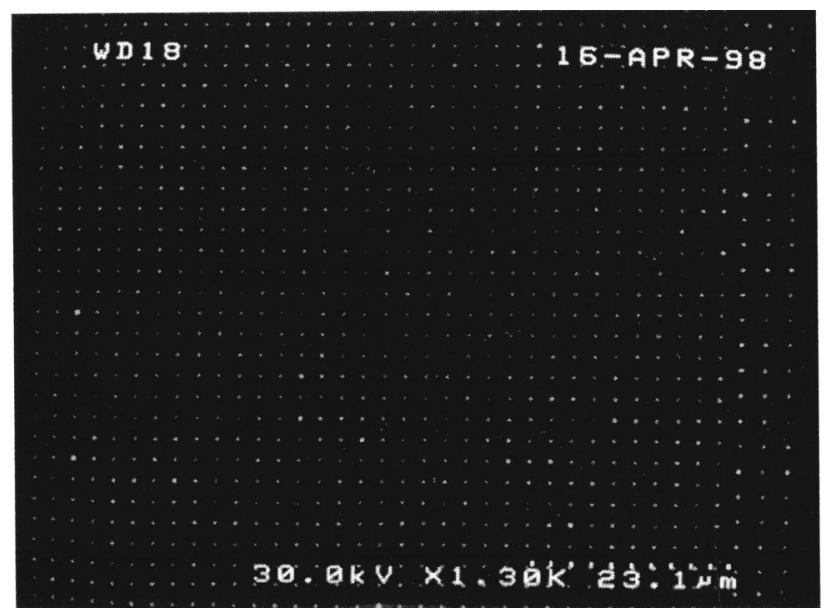

FIG. 3. SEM image of an electroplated Ni dot array of $100 \mathrm{~nm}$ diameter and $2.5 \mu \mathrm{m}$ spacing embedded in an $\mathrm{Al}_{2} \mathrm{O}_{3} / \mathrm{GaAs}$ substrate.

smooth $\mathrm{Al}_{2} \mathrm{O}_{3}$ surface. Atomic force microscopy shows that the columns protrude $20-50 \mathrm{~nm}$ out of the surface, and the area around the columns has a surface roughness of $5 \mathrm{~nm}$. We have also fabricated dot arrays of the same diameter (100 $\mathrm{nm})$ but smaller spacing $(215 \mathrm{~nm})$ using the same technique. However, we chose a 2-2.5 $\mu \mathrm{m}$ column spacing in our structures to match the sizes of the magneto-resistive sensors used in this work. Figures 4(a) and 4(b) show cross-section views of an etched line of $225 \mathrm{~nm}$ width and $1.65 \mu \mathrm{m}$ depth before and after electroplating $\mathrm{Ni}$, respectively. It is believed that the etched holes have similar cross-section profiles as the etched lines, with the exception of slightly smaller diameters and depths, which, however, correspond to similar aspect ratios.

\section{MAGNETIC CHARACTERIZATION}

In order to characterize the magnetic properties of the newly developed patterned media structure, we used both magnetic force microscopy (MFM) ${ }^{7}$ and scanning magnetoresistance microscopy (SMRM).$^{8}$ In the MFM measurement,

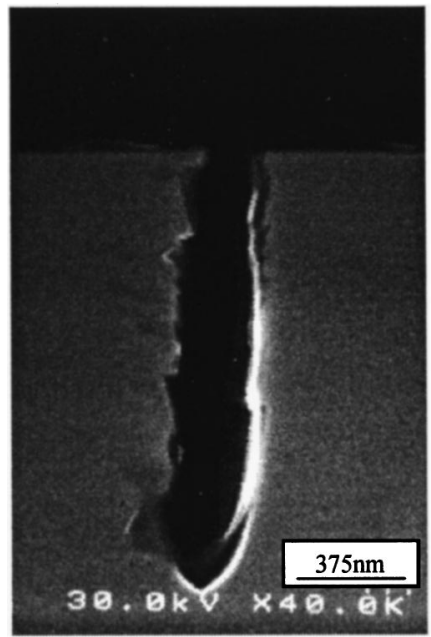

(a)

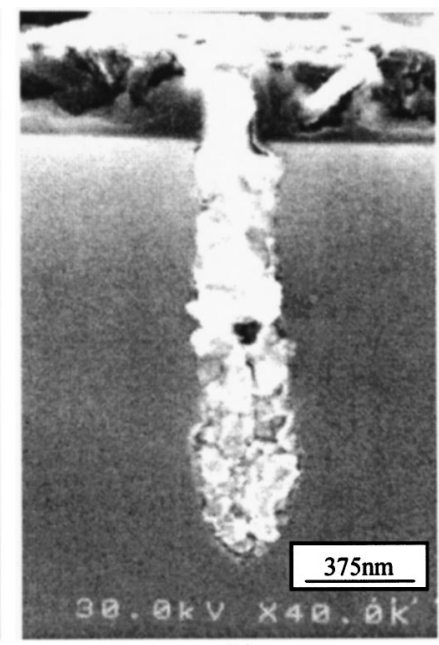

(b)
FIG. 4. Cross-section views of an etched line of $225 \mathrm{~nm}$ width and $1.65 \mu \mathrm{m}$ depth (a) before and (b) after electroplating $\mathrm{Ni}$. 


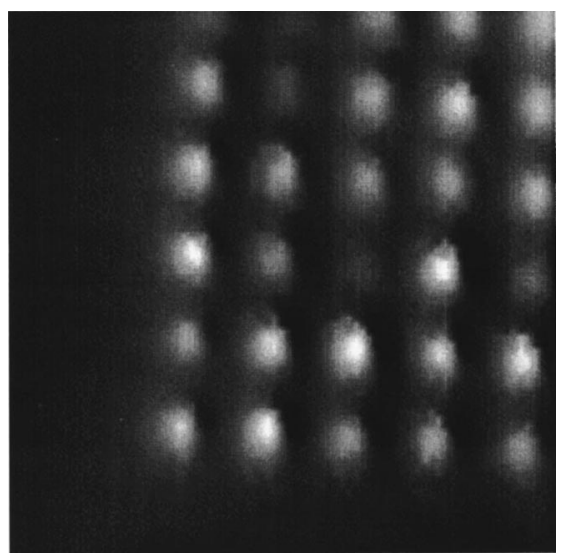

FIG. 5. $12 \times 12 \mu \mathrm{m}$ MFM image of Ni columns of $2 \mu \mathrm{m}$ spacing embedded in an $\mathrm{Al}_{2} \mathrm{O}_{3} / \mathrm{GaAs}$ substrate.

we use a home made MFM utilizing electrochemically etched nickel tips ${ }^{9}$ and a fiber-optic interferometer ${ }^{10}$ as the vibration detector. We focused our investigation on the corner pattern of one of the fabricated arrays in order to compare images from the same columns obtained through the two measurement techniques. Figure 5 shows an MFM image of a $12 \times 12 \mu \mathrm{m}$ scan of plated Ni columns, with diameters of approximately $230 \mathrm{~nm}$, embedded in an $\mathrm{Al}_{2} \mathrm{O}_{3} / \mathrm{GaAs}$ substrate. We believe that the observed nonuniformity in the magnetic strength of the columns is due to the graininess during growth of the plated structures.

In the SMRM technique, ${ }^{8}$ a commercial magnetoresistance (MR) head is raster scanned in contact with the sample and the detected MR voltage is recorded with respect to the position over the sample. In our investigation, we focused on determining the stability of magnetic columns when imaged by this technique. We believe that it is important to demonstrate reliable reading with this type of sensor, since future magnetic storage systems using patterned media are likely to employ a similar read method. Previous work ${ }^{3}$ has shown that the perpendicular component of the magnetic field from the bias current in the MR element was sufficient to switch the column magnetization direction, thus eliminating the possibility of reliably writing the perpendicular patterned media without erasing it through the reading process. The MR signal from the columns embedded in a $\mathrm{SiO}_{2}$ substrate was therefore a "dibit" type signal, and only with the application of the external bias field was a single pole response obtained. ${ }^{3}$

We found that in our current structures, the columns do provide at least a $2 \times$ larger signal when biased with an externally applied magnetic field, which indicates that they relax into a magnetic state that deviates from a uniform magnetization throughout the particle. But more importantly, $85 \%$ of the particles remain magnetized in the direction of the original saturation field, and the MR current is not sufficient to switch them when imaging in zero field. Figures 6(a) -6 (d) show four $12 \times 12 \mu \mathrm{m}$ SMRM images of the same $5 \times 5$ corner array in different external fields, and Fig. 6(e) shows the single line scans that represent the MR voltage signals from each of the images. Figures 6(a) and 6(c) show the different saturation magnetization states of the columns

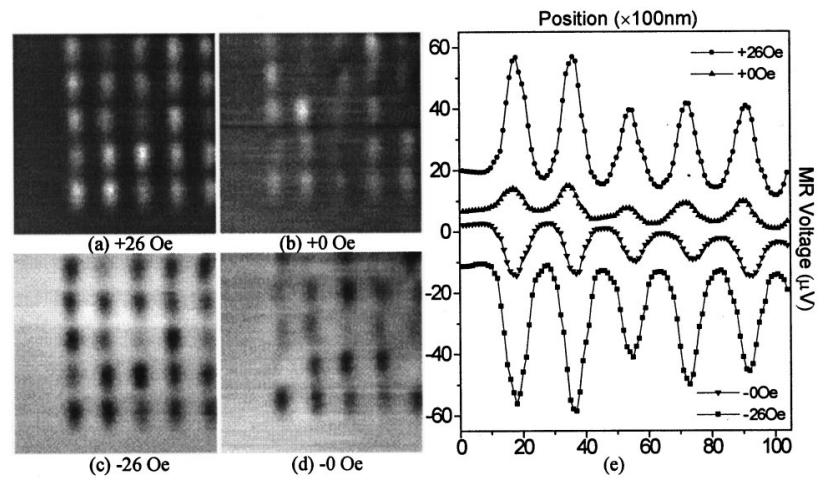

FIG. $6.12 \times 12 \mu \mathrm{m}$ SMRM image of (a) positive saturation field of $+26 \mathrm{Oe}$, (b) 0 Oe positive remanent state, (c) negative saturation field of $-26 \mathrm{Oe}$, (d) 0 Oe negative remanent state, and (e) MR voltage vs position of (a), (b), (c), and (d).

achieved with \pm 26 Oe of applied field, while Figs. 6(b) and 6(d) show the remanent zero field states approached from the positive and negative saturation fields respectively. An important comparison is to be made between images of Figs. 6(b) and 6(d). Even though a few columns provide almost no contrast in zero field, most of the columns still provide large signals and are oriented in the "correct" direction. In the SMRM scans in zero field, we always obtain the single pole response and have not observed a dibit type response, which would have indicated a low switching field magnetic particle. The increase in $H_{c}$ of the individual columns is critical for further improvement in the stability of such media, where the written information must be read nondestructively. We find the width of the column MR response to be $600-700 \mathrm{~nm}$, which is much larger than the column diameter. Such a wide response from a single column has been observed previously. ${ }^{3}$ To our knowledge, the spatial MR response of a perpendicularly magnetized cylinder has not been modeled, and we are currently doing a theoretical investigation of this experimental observation.

\section{ACKNOWLEDGMENTS}

The authors gratefully acknowledge C. C. Cheng and O. J. Painter for many helpful discussions. They thank F. Spada, R. O'Barr, S. Y. Yamamoto, M. Re, and V. Prabhakaran for helpful suggestions, J. F. Smyth for providing them with the MR heads, and R. K. Lee for the growth of the MBE wafers. This work was funded by the National Science Foundation and the Army Research Office.

${ }^{1}$ W. Xu, J. Wong, C. C. Cheng, R. Johnson, and A. Scherer, J. Vac. Sci. Technol. B 13, 2372 (1995).

${ }^{2}$ P. R. Krauss and S. Y. Chou, J. Vac. Sci. Technol. B 13, 2850 (1995).

${ }^{3}$ S. Y. Yamamoto, R. O'Barr, S. Schultz, and A. Scherer, IEEE Trans. Magn. 33, 3016 (1997).

${ }^{4}$ O. J. Painter, C. C. Cheng, and A. Scherer (unpublished).

${ }^{5}$ K. D. Choquette et al., IEEE J. Sel. Top. Quantum Electron. 3, 916 (1997).

${ }^{6}$ R. O'Barr, S. Y. Yamamoto, S. Schultz, W. Xu, and A. Scherer, J. Appl. Phys. 81, 4730 (1997).

${ }^{7}$ Y. Martin and H. K. Wickramasinghe, Appl. Phys. Lett. 50, 1455 (1987).

${ }^{8}$ S. Y. Yamamoto and S. Schultz, Appl. Phys. Lett. 69, 3263 (1996).

${ }^{9}$ D. Rugar, H. J. Mamin, P. Guethner, S. E. Lambert, J. E. Stern, I. McFadyen, and T. Yogy, J. Appl. Phys. 68, 1169 (1990).

${ }^{10}$ D. Rugar, H. J. Mamin, and P. Guethner, Appl. Phys. Lett. 55, 25 (1989). 